\title{
EVOLUTION OF MICROSTRUCTURE DURING SOLIDIFICATION OF Al-Si CAST ALLOYS
}

\author{
Sumanth Shankar ${ }^{1}$, Diran Apelian² and Makhlouf M.Makhlouf ${ }^{3}$ \\ ${ }^{1}$ Post Doctoral Fellow, ${ }^{2}$ Howmet Professor of Eng. and Director of the Metal Processing Institute (MPI), ${ }^{3}$ Professor \\ of Mechanical Eng. and Director of the Advanced Casting Research Center (ACRC) \\ Metal Processing Institute (MPI) \\ Worcester Polytechnic Institute (WPI) \\ Worcester, MA 01609
}

\begin{abstract}
The continuing quest for aluminum castings with enhanced mechanical and physical properties for applications in the automotive and aerospace industries has intensified the interest in aluminum - silicon alloys. Consequently, in the past decade, one of the main research emphases at the Advanced Casting Research Center has been the understanding, quantification, and tailoring of the microstructure of these casting alloys to obtain alloys with better casting characteristics and overall service performance. There are two important stages of microstructure development during the solidification of Al-Si alloys. These are nucleation of the primary phase at, or slightly below, the liquidus temperature of the alloy, and microstructure development during freezing in the mushy (solid + liquid) zone. Microstructural evolution throughout solidification has been established by the use of the advanced microscopic tools that are described in this presentation.
\end{abstract}

Several research efforts have been dedicated to characterizing alloy microstructure in the liquid plus solid (two phase) temperature range. When rheocasting was first discovered, it was believed that one had to break up the dendritic structure during the freezing process either by mechanical stirring or by magneto-hydrodynamic forces in order to obtain a cellular structure. In the recent past, we have discovered that one may obtain a refined SSM (semisolid metal) structure without breaking up the dendritic structure, but rather by creating an environment where copious nucleation can occur near the liquidus temperature of the alloy, and with limited growth of the formed nuclei. Essentially, SSM structures develop by controlling the nucleation and growth processes during the early stages of freezing. A thorough understanding of the fundamental mechanisms responsible for the nucleation and evolution of the primary phase in hypoeutectic Al-Si alloys has led to the development of a continuous slurry on demand process, named the Continuous Rheo-conversion Process (CRP). In this novel casting technique, the resultant morphology of the primary phase has equiaxed grains rather than the typical dendritic structure as shown in Figure 1. This change in primary phase morphology accounts to a large extent for the observed improvements in properties and performance of rheo-cast Al-Si alloy components over their conventionally cast counterparts.

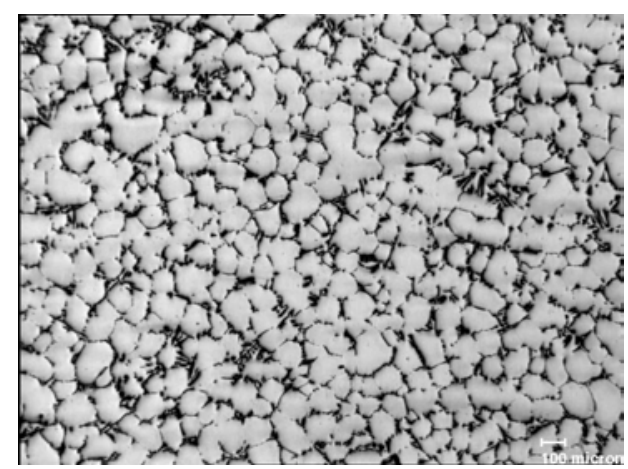

(a)

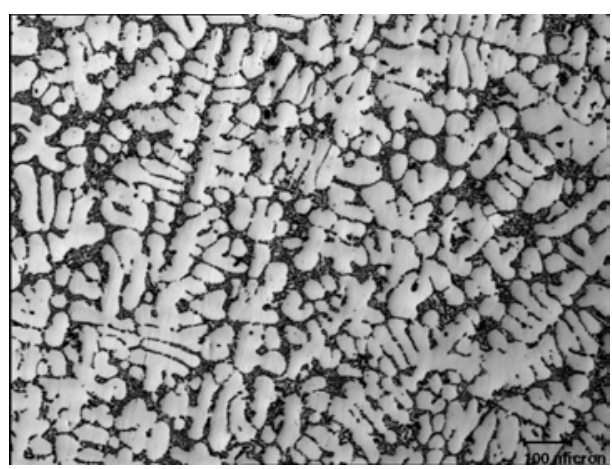

(b)

Figure 1. Comparison of Al-Si alloy cast (a) using the CRP and (b) using a permanent mold. The CRP as-cast microstructure is better than the permanent mold structure for semi-solid processing. 
Understanding the evolution of the eutectic microstructure and the effect of the various alloying elements on the morphology of the Al-Si eutectic is critical to designing castings with better properties. Property detractors such as porosity, microporosity, and defects caused by poor feedability, and intermetallic particle formation, morphology and constituency are greatly influenced by the freezing characteristics of the eutectic phase. In this research, interrupted quench experiments of various Al-Si alloys were performed and the resulting microstructures were characterized using optical, and scanning (SEM) and transmission electron microscopy (TEM). Figure 2 illustrates the capacity of interrupted quench experiments coupled with scanning electron microscopy to provide information regarding the nucleation and growth of the eutectic phase in Al-Si foundry alloys. Conventional TEM sample preparation techniques, such as ion milling and electropolishing, consistently failed to produce samples of cast Al-Si alloys that are of acceptable quality for transmission electron microscopy (TEM) and electron diffraction studies. Consequently, we introduced the focused ion beam (FIB) milling technique as a viable method for producing high quality TEM samples of cast aluminum-silicon alloys. Figures 3 and 4 show a comparison of microstructures using SEM and TEM of Al-7wt.\% Si and Al-7wt.\%Si-0.022wt.\% Sr air cooled castings. Analyses of these microstructures have shown conclusive evidences for the mechanism of nucleation and growth of the eutectic phases. Addition of trace elements such as $\mathrm{Na}$ and $\mathrm{Sr}$ not only modifies the morphology of the Si but also grain refines the eutectic aluminum phase. The mechanism behind these modifications has also been established through extensive metallographic analysis.

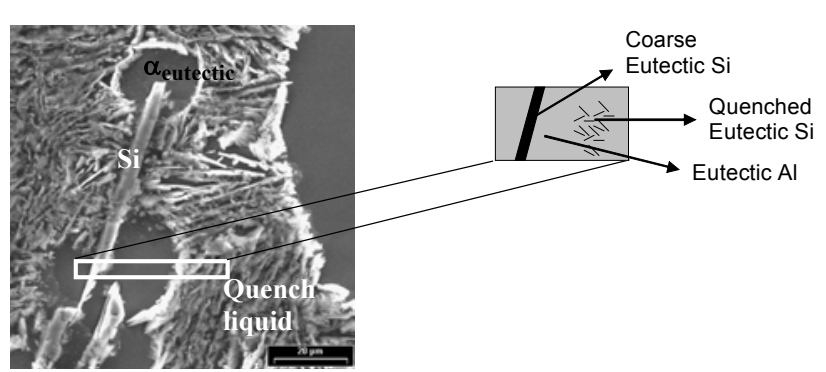

(a)

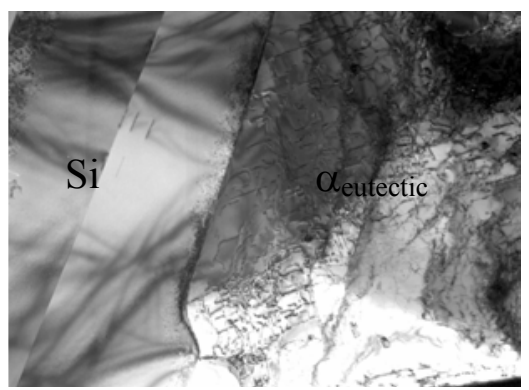

(b)

Figure 2. Microstructures of aluminum eutectic grains nucleating on silicon flakes during the solidification of an Al-Si foundry alloy. (a) SEM micrograph and (b) TEM micrograph. The sample was obtained by quenching after $20 \%$ of the eutectic reaction was completed. Also shown is the region where TEM samples were milled by FIB.

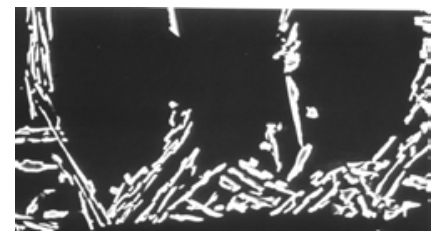

(a)

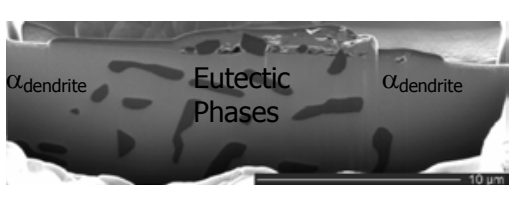

(b)

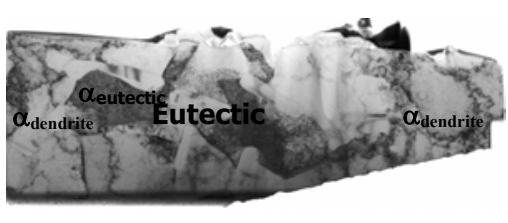

(c)

Figure 3. Comparison of microstructures from (a) SEM, (b) SEM after FIB milling, and (c) TEM Al-7wt.\%Si (air cooled) alloy.

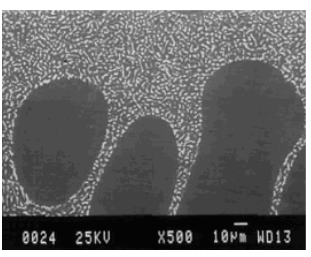

(a)

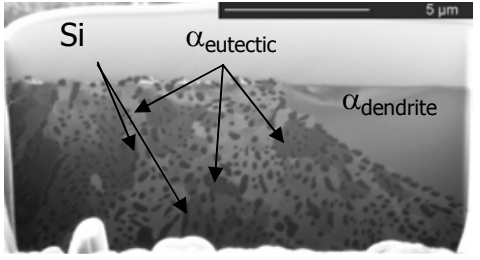

(b)

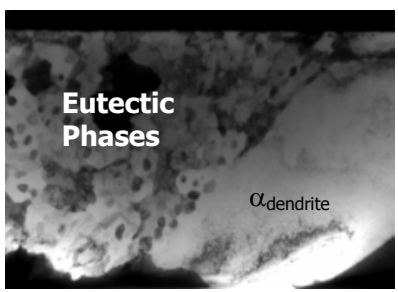

(c)

Figure 4. Comparison of microstructures from (a) SEM, (b) SEM after FIB milling, and (c) TEM Al-7wt.\%Si (air cooled) alloy with $0.022 \mathrm{wt} . \% \mathrm{Sr}$. 